\title{
Editorial: Ethnopharmacology in Central and Eastern Europe in the Context of Global Research Developments
}

\author{
Devesh Tewari ${ }^{1}$, Judit Hohmann ${ }^{2}$, Anna K. Kiss ${ }^{3}$, Judith M. Rollinger ${ }^{4}$ and \\ Atanas G. Atanasov ${ }^{4,5,6 *}$ \\ ${ }^{1}$ Department of Pharmacognosy, School of Pharmaceutical Sciences, Lovely Professional University, Phagwara, India, \\ ${ }^{2}$ Department of Pharmacognosy, University of Szeged, Szeged, Hungary, ${ }^{3}$ Department of Pharmacognosy and Molecular \\ Basis of Phytotherapy, Medical University of Warsaw, Warsaw, Poland, ${ }^{4}$ Department of Pharmacognosy, University of Vienna, \\ Vienna, Austria, ${ }^{5}$ Institute of Genetics and Animal Breeding of the Polish Academy of Sciences, Magdalenka, Poland, \\ ${ }^{6}$ GLOBE Program Association (GLOBE-PA), Grandville, MI, United States
}

Keywords: pharmacognosy, phytochemistiy, pharmacology, ethnobotany, ethnopharmacology

OPEN ACCESS

Edited by:

Javier Echeverria,

Universidad de Santiago de Chile,

Chile

Reviewed by:

Michael Heinrich,

UCL School of Pharmacy,

United Kingdom

${ }^{*}$ Correspondence:

Atanas G. Atanasov

a.atanasov.mailbox@gmail.com

Specialty section:

This article was submitted to

Ethnopharmacology,

a section of the journal

Frontiers in Pharmacology

Received: 30 January 2019

Accepted: 19 March 2019

Published: 09 April 2019

Citation:

Tewari D, Hohmann J, Kiss AK,

Rollinger JM and Atanasov AG (2019)

Editorial: Ethnopharmacology in

Central and Eastern Europe in the

Context of Global Research

Developments.

Front. Pharmacol. 10:341.

doi: 10.3389/fphar.2019.00341
Editorial on the Research Topic

Ethnopharmacology in Central and Eastern Europe in the Context of Global Research Developments

Historically, medicinal plants have been a key element of healthcare, and are still widely used as alternative and complementary therapy (mainly in developed countries) or as a primary treatment (in most developing countries). Moreover, many of the modern pharmaceuticals were developed from molecules extracted from natural sources, and medicinal plants still today represent an important pool for the identification of novel drug leads.

The ethnopharmacological tradition in Central and Eastern Europe has a great historical significance and large parts of the Western world's knowledge for therapeutic properties of medicinal plants has its roots in the Greek and Roman cultures (e.g., with a reference to the influential works of Dioscorides, Pliny the Elder, and Galen). The German-speaking Central European areas also have played very important roles, with some influential medieval herbal book editions such as the Mainz Herbal (Herbarius Moguntinus, 1484) and The German Herbal (1485). Moreover, at the beginning of the nineteenth century modern drug discovery from plants started to played a key role in Central Europe, most notably the work of the German apothecary assistant Friedrich Sertürner, who isolated an analgesic and sleep-inducing agent from opium that was named morphium (morphine) after the Greek god of dreams, Morpheus. At the same time, it should be noted that on many occasions the rich ethnopharmacological knowledge of some East European countries and Russia remained localized and did not find its way to integrate with the Western world of herbal therapy traditions. The focus of this Research Topic is to make a special contribution to ethnopharmacology rooted in Central and Eastern Europe, on the context of global research developments in the area of ethnopharmacology, phytochemistry, molecular pharmacology of natural products, nutrigenomics, and other related disciplines. This special issue consists of 24 articles covering diverse topics related to the listed research fields.

Advances in screening methods and analytical equipment, increasing number of targets available for testing, and improved possibilities for optimization of natural leads using synthetic modification 
strategies substantially improved the process of modern drug discovery from natural sources (Atanasov et al., 2015). Hypericum is a well-known genus that is used for its medicinal properties. Tocci et al. evaluated five Hypericum species for their polyphenolic content, toxicological safety, and antifungal potential against some sensitive and drug resistant clinical fungal isolates. The authors have identified 52 compounds by LC-MS analysis and showed that $H$. hircinum subsp. majus infusion exerted broad antifungal activity against Aspergillus, Penicillium, and non-albicans Candida strains (Tocci et al.).

Ethnobotany has played an important role in new drug development and drug discovery (Kayser, 2018). The importance of the traditional use of medicinal plants on the Greek islands of the North Aegean Region was summarized in a Brief Research Report Article by Axiotis et al. In this report the authors present 109 wild plants from 52 families, and enlist their therapeutic uses, including uses in galenic preparations which were reported by local medical doctors and pharmacists (Axiotis et al.).

Medicinal plants and natural products are of potential importance in several age-related disorders, including memory impairment and dementia (Tewari et al., 2018). In this research topic Dimpfel et al. assessed the biological activity of some commercial extracts of the underground parts of Rhodiola rosea L. and its analytical markers, salidroside and rosavin, using longterm potentiation of synaptic transmission in hippocampus slices (a synaptic model of memory).

Loeser et al. evaluated the effect of Casperome ${ }^{\circledR}$, an orally bioavailable soy lecithin-based formulation of standardized frankincense extract on various systemic parameters and organ damages that were induced by severe systemic inflammation caused by intraperitoneal administration of lipopolysaccharides (a murine model of sepsis). The study revealed that the extract possesses anti-inflammatory, anti-oxidant, and hepatoprotective effects (Loeser et al.).

Zielińska et al. review older and recent data on pharmacology, phytochemistry, and clinical studies of Chelidonium majus L. along with controversies about this herb, its safety, and drug quality issues.

The study of Wozniak et al. evaluated the effects of phytochemically characterized extracts of various parts of traditionally used Syringa vulgaris L. (common lilac) on the pro-inflammatory functions of neutrophils. The authors also isolated active compounds, including neooleuropein from the extracts using bioassay-guided fractionation. It was found that neooleuropein acts through inhibition of cytokine production by attenuation of the MAP kinase pathways (Woźniak et al.).

Smooth muscle cells are a prominent target of diverse natural products with different bioactivities (Hnatyszyn et al., 2003; Uhrin et al., 2018). Sándor et al. assessed the relaxant effects or contracting activity of the hydroethanolic extract, fractions, phytoconstituents, and essential oil of Roman chamomile on smooth muscle preparations. The authors found that the essential oil of the plant possessed a good smooth muscle-relaxant effect (Sándor et al.).

Identification of the molecular mechanism of action of promising natural products is a vital step toward their clinical application (Butler, 2008; Tewari et al., 2018). In a mechanistic study, Celińska-Janowicz et al. studied the apoptosisinducing mechanisms of selected propolis components in tongue squamous cell carcinoma cells (CAL-27). In another work, Grienke et al. isolated several flavonoids and rare sesquiterpene lactones from the ethanolic extract of Centaurea ragusina L., a plant species endemic to Croatia. For the first time they identified ragusinin, and hemistepsin $\mathrm{A}$ from this genus. The authors further studied the mechanism underlining the ragusinin activity in cancer cells (Grienke et al.).

In their review article Kozlowska et al. summarize the herbal medicines used in the Beskid mountain ranges, which are located south of Krakow and Lviv, two important medieval centers of apothecary tradition in the area. In another interesting review Kunnumakkara et al. summarize potential benefits, traditional uses, chemistry, biological activities, and clinical trials of "guggul" from Commiphora and Boswellia.

Berberine is an important phytoconstituent of plants utilized in different traditional medicine systems, including the Chinese system of traditional medicine (Huang et al., 2011). In their work "Berberine: Botanical Occurrence, Traditional Uses, Extraction Methods, and Relevance in Cardiovascular, Metabolic, Hepatic, and Renal Disorders" Neag et al. review diverse biomedical information on berberine, including several chromatographic techniques for berberine extraction and quantification.

There is great interest of the scientific community toward the health benefits of food items used in the Mediterranean diet, which has a variety of health benefits (Sofi et al., 2010). Wang et al. review recent studies on pomegranate, mainly focusing on vasculoprotective effects attributed to diverse pomegranate phytoconstituents, including tannins, especially ellagitannins, and ellagic acid, among other compounds. Lycopene is a carotenoid which is responsible for different pigmentation in tomatoes, grapefruit, and watermelon, and it represents a compound with a potent antioxidant action (Horvitz et al., 2004). In their work, Mozos et al. review mechanisms of action of lycopene on the vascular system considering substantial epidemiological and experimental data, and clinical studies.

Breast cancer is the most common cancer in women and represents a global health burden both in the developed and developing countries. Hong et al. study apigenin and luteolin to better understand their mechanism of action in the context of breast cancer intravasation of the lymphatic barrier. Both of these natural compounds were evaluated in a three-dimensional assay system containing MDA-MB231 breast cancer spheroids and immortalized lymph endothelial cell monolayers (Hong et al.)

Toiu et al. evaluated the chemical composition, antimicrobial, antioxidant, and in vivo anti-inflammatory activity of various extracts of the aerial parts of an important plant species with traditional use in Romanian, Ajuga laxmannii (Murray) Benth.

In their work, Shikov et al. review 70 wild plant species that are referenced in the 11th edition of the State Pharmacopeia of the USSR and also discuss their health food value, mainly based on published Russian literature.

Marchelak et al. present a comprehensive study focused on extracts of the flowers of Prunus spinosa L. The authors 
profiled the phenolic contents of the extracts through UHPLC-PDA-ESI-MS ${ }^{3}$, and also studied several biological effects with relevance in the context of cardiovascular disease (Marchelak et al.).

Chowdhury et al. explore the anti-proliferative and antimetastatic potential of various extracts of the medicinal plant colocynth. The authors have found that ethanol and acetone extracts of colocynth fruit pulp exhibit promising activity in different cancer cell types, inhibiting viability, and cell migration in association with the modulation of the expression of diverse relevant genes and cellular pathways (Chowdhury et al.).

Ononis natrix is a member of the Fabaceae family and is a lessexplored plant species. In their work, Yerlikaya et al. give new insight on the chemical composition and biological activities of Ononis natrix subsp. hispanica.

Wang et al. identify and characterize the natural product falcarindiol as a novel inducer of macrophage cholesterol efflux, a process with a protective effect in atherosclerosis.

In their research paper, Raclariu et al. establish DNA metabarcoding and HPLC-MS techniques for the authentication of Veronica officinalis L. herbal products. The authors advocate for the use of an integrative approach for the detection of adulteration and substitution in the herbal products (Raclariu et al.).

López et al. study the effects of lavender essential oil on pharmacological targets that are involved in anti-depressive and anxiolytic effects, as well as on in vitro models of neurotoxicity.

\section{REFERENCES}

Atanasov, A. G., Waltenberger, B., Pferschy-Wenzig, E. M., Linder, T., Wawrosch, C., Uhrin, P., et al. (2015). Discovery and resupply of pharmacologically active plant-derived natural products: a review. Biotechnol. Adv. 33, 1582-1614. doi: 10.1016/j.biotechadv.2015.08.001

Butler, M. S. (2008). Natural products to drugs: natural product-derived compounds in clinical trials. Nat. Prod. Rep. 25, 475-516. doi: 10.1039/ b514294f

Hnatyszyn, O., Moscatelli, V., Garcia, J., Rondina, R., Costa, M., Arranz, C., et al. (2003). Argentinian plant extracts with relaxant effect on the smooth muscle of the corpus cavernosum of guinea pig. Phytomedicine 10, 669-674. doi: 10.1078/0944-7113-00261

Horvitz, M. A., Simon, P. W., and Tanumihardjo, S. A. (2004). Lycopene and $\beta$-carotene are bioavailable from lycopene 'red' carrots in humans. Eur. J. Clin. Nutr. 58, 803-811. doi: 10.1038/sj.ejcn. 1601880

Huang, Z. J., Zeng, Y., Lan, P., Sun, P. H., and Chen, W. M. (2011). Advances in structural modifications and biological activities of berberine: an active compound in traditional Chinese medicine. Mini Rev. Med. Chem. 11, 1122-1129. doi: 10.2174/138955711797655362

Kayser, O. (2018). Ethnobotany and medicinal plant biotechnology: from tradition to modern aspects of drug development. Planta Med. 84, 834-838. doi: 10.1055/a-0631-3876
Lewenhofer et al. performed an activity-guided identification of active principles from Scrophularia lucida L. using cancer cell models. The authors identified 14 substances including some very rare iridoids, like scrovalentinoside or koelzioside, and flavonoids such as nepitrin and homoplantaginin (Lewenhofer et al..)

In conclusion, this Research Topic includes a wide range of interdisciplinary research work contributing to the ultimate goals of augmenting the existing knowledge and fostering the scientific field of ethnopharmacology in Central and Eastern Europe, as well as globally. This research topic successfully gathered comprehensive interdisciplinary information in the field of ethnopharmacology from the field surveys to standardization and elucidation of molecular mechanisms of action of natural products. Moreover, with consideration of the existing challenges in the field of drug discovery form medicinal plants, this research topic provides up-to-date snapshot of current knowledge and paves the way for further chemical and biological assessment of diverse natural compounds with potential for future therapeutic development. We hope that this compendium will further inspire scientists from different research fields to make use of the gathered traditional medical knowledge in the search for superior future remedies from nature.

\section{AUTHOR CONTRIBUTIONS}

All authors listed have made a substantial, direct and intellectual contribution to the work, and approved it for publication.

Sofi, F., Abbate, R., Gensini, G. F., and Casini, A. (2010). Accruing evidence on benefits of adherence to the Mediterranean diet on health: an updated systematic review and meta-analysis. Am. J. Clin. Nutr. 92, 1189-1196. doi: 10.3945/ajcn.2010.29673

Tewari, D., Stankiewicz, A. M., Mocan, A., Sah, A. N., Tzvetkov, N. T., Huminiecki, L., et al. (2018). Ethnopharmacological approaches for dementia therapy and significance of natural products and herbal drugs. Front. Aging Neurosci. 10:3. doi: 10.3389/fnagi.2018.00003

Uhrin, P., Wang, D., Mocan, A., Waltenberger, B., Breuss, J. M., Tewari, D., et al. (2018). Vascular smooth muscle cell proliferation as a therapeutic target. Part 2: natural products inhibiting proliferation. Biotechnol. Adv. 36:1608-21. doi: 10.1016/j.biotechadv.2018.04.002

Conflict of Interest Statement: The authors declare that the research was conducted in the absence of any commercial or financial relationships that could be construed as a potential conflict of interest.

Copyright (C) 2019 Tewari, Hohmann, Kiss, Rollinger and Atanasov. This is an openaccess article distributed under the terms of the Creative Commons Attribution License (CC BY). The use, distribution or reproduction in other forums is permitted, provided the original author(s) and the copyright owner(s) are credited and that the original publication in this journal is cited, in accordance with accepted academic practice. No use, distribution or reproduction is permitted which does not comply with these terms. 